\title{
Abordaje clínico y manejo de la depresión posparto
}

\author{
Clinical approach and management of postpartum depression
}

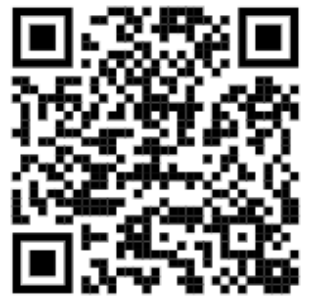

${ }^{1}$ Médico general, graduada de la Universidad de Ciencias Médicas (UCIMED), código médico:14634

solismariana92@hotmail.com

${ }^{2}$ Médico general, graduada de la Universidad Latina de Costa Rica (U. Latina), código médico: 14780

analupin1@gmail.com

${ }^{3}$ Médico general, graduada de la Universidad Latina de Costa Rica (U. Latina), código médico: 15051

joselyn chacon65@hotmail.com
${ }^{1}$ Dra. Mariana Solís Solano Investigadora Independiente, San José, Costa Rica

(D) https://orcid.org/0000-0002-5380-1712

${ }^{2}$ Dra. Ana Lucia Pineda Mejía Investigadora Independiente, San José, Costa Rica (D) https://orcid.org/0000-0002-8925-9611

${ }^{3}$ Dra. Joselyn Chacón Madrigal Investigadora Independiente, San José, Costa Rica (D) https://orcid.org/0000-0002-1075-4899

RECIBIDO

CORREGIDO

ACEPTADO

06/04/2019

$17 / 04 / 2019$

$01 / 05 / 2019$

\section{RESUMEN}

El puerperio o posparto, es el periodo que se da inmediatamente posterior al parto hasta las seis semanas generalmente, este tiempo es de suma importancia debido a que se da la transición de los cambios fisiológicos ocurridos durante el embarazo al estado fisiológico previo. Durante este período ocurren varios procesos de adaptación a nivel social, emocional y hormonal en la mujer, que influyen directamente a nivel psicológico. De ahí la importancia de detectar a tiempo los posibles factores de riesgo de una depresión posparto, su pronto diagnóstico, su correcto manejo y tratamiento; para así evitar futuras complicaciones que involucren trastornos mentales en la madre, en el proceso de lactancia, vinculación entre la madre y el producto, así como en el futuro desarrollo del niño.

PALABRAS CLAVE: puerperio; depresión posparto; psicoterapia; antidepresivos.

\section{ABSTRACT}

The puerperium or postpartum, is the period that occurs immediately after childbirth until six weeks generally, this time have great importance because of the transition that occurs from the physiological changes during pregnancy to the previous physiological state. During this period several processes of adaptation take place at a social, emotional and hormonal level in women, which influence directly in the psychological state. Hence the importance of to detect 
in time the possible risk factors of a postpartum depression, the early diagnosis, the correct handling and treatment; in order to avoid future complications that involve mental disorders in the mother, the process of breastfeeding, attachment between the mother and the product, as the future development of the child.

KEYWORDS: puerperium; postpartum depression; psychotherapy; antidepressive agents.

\section{INTRODUCCIÓN}

La depresión posparto ocurre entre un $15 \%$ y $20 \%$ en mujeres, esto resulta en 600,000 a 800,000 casos aproximados de depresión posparto al año, es una de las complicaciones más comunes en el período posparto (1). Dado su alta prevalencia y múltiples consecuencias tanto en la madre como el niño a corto y largo plazo; debido a un diagnóstico tardío e manejo inadecuado.

Este artículo se centra en la identificación temprana de factores de riesgo, para así poder instaurar conductas de vigilancia, prevención y por ende facilitar la pronta detección de las manifestaciones clínicas, que en un inicio pueden ser de aparición insidiosa, pero su permanencia las lleva a ser catalogadas como una depresión como tal. Esto con el objetivo de instaurar un manejo terapéutico de acuerdo a la severidad de la depresión, tomando en cuenta a la psicoterapia como pilar en todos los niveles de gravedad clínica, con adaptaciones de la misma o asociado a farmacoterapia, según el caso estudiado.

\section{GENERALIDADES}

El puerperio o periodo posparto, se define como el período posterior al parto de seis semanas aproximadamente (2), es el tiempo de adaptación después del parto cuando se revierten los cambios anatómicos, fisiológicos y el cuerpo de la madre regresa al estado previo. Por lo que durante este periodo ocurren diversos cambios a nivel neuroendocrino y psicosocial; pudiendo así generar en la madre una depresión posparto; que se define como un episodio de depresión mayor que es asociado temporalmente al parto (3), con un inicio dentro de los 12 meses siguientes al parto y que los síntomas deben estar presentes casi a diario durante al menos dos semanas (4). Los síntomas que se incluyen en la depresión mayor postparto abarcan desde; sentimiento de tristeza, sin esperanza durante todo el día, sensación de pérdida del interés o placer, disminución o pérdida del apetito, insomnio o hipersomnia, percepción de inquietud 0 enlentecimiento en sus actividades, fatiga, pérdida de energía durante el día, desatención de las actividades importantes, ideas suicidas, pensamientos de muerte sin un plan estructurado. Estableciéndose que todos estos síntomas anteriores, causen una disfunción social 0 de relaciones interpersonales de las madres postparto; cabe recalcar que las mujeres con criterios de otros trastornos especificados o no especificados del espectro de la esquizofrenia $y$ otros trastornos psicóticos, no se incluyen en esta revisión, debido a que no se encuentran 
dentro del diagnóstico de depresión mayor postparto (4).

\section{FACTORES DE RIESGO}

El mayor factor de riesgo para la depresión posparto es el de antecedente de este trastorno previamente al embarazo (2), en mujeres que han sufrido depresión anteriormente y también en quienes carecen de mecanismos de apoyo eficaces; pudiendo manifestarse esta patología con síntomas de mayor intensidad y por ende el riesgo de sufrir consecuencias más graves (4).

Según el DSM-V se evidencia que en: "Estudios prospectivos han demostrado que los síntomas del estado de ánimo y de ansiedad durante el embarazo, así como el desánimo puerperal aumentan el riesgo de un episodio de depresión mayor después del parto".

Además se ha observado que las mujeres que cursan con episodios de depresión mayor en el periparto, con frecuencia sufren ansiedad grave e incluso ataques de pánico asociados a este trastorno en el posparto (4).

Respecto a otros trastornos psiquiátricos relacionados, el riesgo de episodios posparto con características psicóticas es especialmente mayor en mujeres con antecedentes de trastornos del ánimo en el puerperio, pero también se eleva en pacientes con enfermedades mentales no diagnosticadas previamente $y$ en aquellas con antecedentes de un trastorno depresivo o bipolar (en especial tipo I) y al asociar antecedentes heredofamiliares de enfermedades bipolares. También se considera que una mujer ha tenido un episodio posparto con características psicóticas, el riesgo de recurrencia es del $30 \%$ al $50 \%$ de episodios con cada parto posterior (4).

Además, se incluyen como otros antecedentes de riesgo: intentos de suicidio previos, intentos de suicidio en familiares cercanos, historial de comportamientos impulsivos, antecedentes de hospitalización previos y violencia intrafamiliar durante la gestación o en el último año, drogadicción, alejamiento social o de su núcleo familiar, conflictos de pareja y problemas legales (5).

También se ha observado como factores que aumentan el riesgo: madres que lastiman a sus hijos, edad joven, poco o ningún control prenatal, no tener soporte o apoyo familiar, ni planes a futuro de cuido para el infante $(6,7)$.

\section{DIAGNÓSTICO}

Consideraciones para el diagnóstico de depresión mayor en el comienzo del postparto, según el DSM-V: Se deben presentar 5 o más de los siguientes síntomas (que pueden estar presentes durante todo el día, casi todos los días, por al menos 2 semanas).

1) Humor depresivo: se presenta comúnmente 4-5 días después del parto conocido como "babyblues", que puede durar de horas a días pero no sobrepasa las 2 semanas, si sobrepasa las 2 semanas se conoce entonces como depresión posparto.

2) Falta de interés 0 placer en actividades: es infrecuente que se presente después del nacimiento, cabe recalcar que se debe prestar atención ante la presencia de anhedonia, que es sugestivo de depresión postparto. 
3) Alteración del sueño: es común debido a cuidados que se deben brindar al recién nacido. Pero la imposibilidad de conciliar el sueño cuando el recién nacido duerme o incapacidad de cuidar de él por consecuencia de hipersomnia, se debe sospechar de depresión postparto.

4) Pérdida / falta de energía: es común a causa de privación del sueño por cuido del recién nacido, pero se debe sospechar si continua a pesar del adecuado sueño y siestas de la madre.

5) Agitación 0 retraso psicomotor: sospechar si hay enlentecimiento al hablar o incapacidad de permanecer sentada en un mismo lugar.

6) Sentimientos excesivos de culpa: es infrecuente posterior al parto, por lo que se debe sospechar de depresión posparto ante sentimientos de fracaso, decepción o culpa.

7) Disminución de la concentración: frecuentemente asociado por falta de sueño, por otro lado si madre concilia el sueño y presenta inhabilidad de toma de decisiones y pérdida de continuidad del pensamiento, sospechar depresión postparto.

8) Ideas suicidas: pensamientos como, "desearía no despertar más "o "mi bebe estaría mucho mejor sin mí", intentos, como también deseos o plan de terminar su vida $(8,4)$.

\section{DIAGNÓSTICO DIFERENCIAL}

El principal diagnóstico diferencial de este trastorno es la tristeza o melancolía posparto, ésta última se caracteriza por síntomas transitorios de depresión, como se mencionó anteriormente (4). También se debe diferenciar del síndrome confusional que sucede en el período posparto y se diferencia por un grado de conciencia o atención fluctuante (4).

Además es importante tomar en cuenta diagnósticos de tipo orgánico, como lo son las alteraciones en metabolismo de hormonas tiroideas (9) y el hipotiroidismo (4), cambios en funciones de neurotransmisores (10) y reducción de la algunas citoquinas inflamatorias, como la interleucina 2 en la deficiencia de hierro, podrían ser causas subyacentes del desarrollo de depresión postparto $(11,12)$. Además, recientemente se ha considerado que la condición de Anemia por Deficiencia de Hierro (IDA), asocia síntomas de alteración de la conducta como cambios cognitivos, cambios emocionales como irritabilidad, apatía, fatiga, síntomas depresivos e hipoactividad (13,14); esto debe ser tomado en consideración, debido a que la prevalencia de esta patología es de un $7.5 \%$ en el embarazo y el retraso en la recuperación de estos depósitos de hierro es frecuentemente experimentado en el período posparto; haciendo considerar esta patología como importante diagnóstico diferencial de la depresión postparto $(9,13)$.

\section{MANEJO Y TRATAMIENTO}

El período postnatal de la madre y su recién nacido juegan un papel fundamental para la implementación de medidas centradas en mejorar la salud de ambos, la necesidad crítica de un chequeo posparto entre el 3er y 6to día poshospitalario es una excelente oportunidad para evaluar la aparición temprana de los síntomas depresivos 
(15). La evaluación inicial de las mujeres posparto con sospecha de la depresión mayor, es similar a la de las pacientes con la misma sospecha, que no estén en un período postparto. La evaluación incluye la historia de trastornos médicos psiquiátricos y generales, el estado mental, el examen físico y se incluyen además, pruebas de laboratorio para descartar una afección médica general que cause un cuadro similar (16).

Una vez identificados los síntomas posparto mencionados anteriormente, el primer paso en el manejo de una mujer con depresión posparto; es una valoración para determinar la gravedad de su depresión y para determinar si representa una amenaza para sí misma o para otros.

Pacientes con síntomas graves (p. ej., ideación suicida con un plan y una intención específicos de comportamiento suicida, o las alucinaciones auditivas) deben ser referidos a un departamento de Urgencias y así obtener una interconsulta psiquiátrica lo antes posible para una mayor evaluación y abordaje necesario e inmediato, así como medidas de protección tanto para madre como para su recién nacido. En casos severos de depresión posparto, especialmente en las madres que están en riesgo de suicidio, su hospitalización puede ser necesario (17).

\section{DIAGNÓSTICO DIFERENCIAL}

\section{DEPRESIÓN POSTPARTO LEVE - MODERADA}

Las pacientes con enfermedades de leve a moderada generalmente no manifiestan comportamientos suicidas 0 evidente deterioro del funcionamiento, y es menos probable que desarrollen complicaciones tales como rasgos psicóticos y características catatónicas (18). Por lo que en la depresión leve a moderada normalmente se puede administrar terapia en pacientes ambulatorios 0 atención hospitalaria parcial durante el día, ya que se sugiere la psicoterapia como tratamiento inicial; este enfoque es coherente con las guías de práctica clínica y es especialmente útil para madres lactantes, que no desean exponer a sus bebés a los antidepresivos $(19,20)$.

\section{- Psicoterapia}

En función de su eficacia demostrada en múltiples ensayos aleatorios en la población general de pacientes con depresión mayor, así como pacientes con depresión posparto se utiliza la psicoterapia para tratar la depresión mayor posparto, generalmente se elige terapia cognitiva conductual o psicoterapia interpersonal. Sin embargo, otros métodos alternativos razonables incluyen: la activación del comportamiento, el asesoramiento no directivo y la psicoterapia psicodinámica como aliados en dicho abordaje (16).

\section{a) Terapia cognitivo - conductual}

En este tipo de terapia se combinan la terapia cognitiva, que está destinada a modificar los pensamientos disfuncionales 0 creencias de la enfermedad y la terapia conductual, la cual está destinada a cambiar las conductas problemáticas que ocurren en respuesta a pensamientos disfuncionales, síntomas depresivos y estímulos ambientales que sufren las madres que padecen de esta enfermedad (21). 
Además, en esta terapia se utiliza como método de intervención a las madres en terapia la activación del comportamiento; con el objetivo de contrarrestar comportamientos de inercia y de evitación; para así promover actividades y comportamientos que sean gratificantes, ayudando a las pacientes a disminuir conductas de evitación y mejorar sus habilidades para resolver problemas (22).

\section{b) Psicoterapia interpersonal}

Este tipo de terapia se centra en mejorar las relaciones interpersonales problemáticas o las circunstancias que están directamente relacionadas con el episodio depresivo actual; estos problemas interpersonales incluyen disputas de roles (por ejemplo, conflictos conyugales) y transiciones de roles (por ejemplo, convertirse en madre) este último es uno de los más importantes, ya que las mujeres experimentan cambios físicos, además de nuevas responsabilidades maternas (23).

\section{c) Asesoramiento no directivo}

Tiene como objetivo ayudar a las pacientes a comprender y aceptar sus sentimientos, valores y comportamiento. El terapeuta actúa como facilitador al alentar a las pacientes a hablar, en lugar de proporcionar explicaciones o interpretaciones (24).

\section{d) Psicoterapia psicodinámica}

Esta pretende mejorar la comprensión de los conflictos repetitivos mediante la identificación de patrones de relaciones, sentimientos y comportamientos; el paciente trabaja para desarrollar estilos de afrontamiento más productivos (mecanismos de defensa) (25).

Aunque la psicoterapia suele administrarse individualmente, algunas psicoterapias (terapia cognitivo conductual y psicoterapia interpersonal) para la depresión posparto se han adaptado con éxito a un formato grupal. La terapia de grupo incluye varios elementos útiles, como desarrollar habilidades de comunicación, normalizar los problemas de uno al recibir apoyo (por ejemplo, consejo, empatía y validación) de otros pacientes que están experimentando problemas similares, reducir el aislamiento social y los sentimientos de soledad, aumentando el sentido de pertenencia y compañerismo, aprendizaje a través del modelado de otros (26).

Además la psicoterapia, suele ser limitada en el tiempo (por ejemplo de 6 a 12 sesiones) independientemente si la falta de respuesta persiste durante las fases intermedias del tratamiento. Sin embargo, no se conoce la duración óptima del tratamiento, y las intervenciones relativamente cortas pueden ser preferibles para las nuevas madres que sienten que carecen del tiempo y la energía para participar en tratamientos más prolongados (27).

Para las pacientes que reciben tratamiento con terapia cognitivaconductual o psicoterapia interpersonal que logran una respuesta parcial $(25 \%$ al $49 \%$ ), se recomienda aumentar el número total de sesiones (de 12 a 16 sesiones) y la frecuencia del tratamiento; debido a que se ha observado que recibir la terapia tiene más beneficios aunque no se evidencie una remisión completa de los síntomas, tomando en cuenta que estas pacientes siguen una línea de tratamiento no farmacológica y por ende no se exponen ante los efectos adversos de los medicamentos antidepresivos (22). 
Para las pacientes que reciben tratamiento con terapia cognitivo conductual o psicoterapia interpersonal y logran solo una respuesta mínima (mejoría de menos del 25\%), se recomienda cambiar a otra psicoterapia. Otras alternativas de psicoterapia razonables para la depresión posparto resistente a la terapia, incluyen la activación conductual, el asesoramiento no directivo, la psicoterapia psicodinámica o la adición de fármacos antidepresivos, ante la persistencia de una mínima respuesta o resistencia pese a los cambios mencionados anteriormente (22).

Respecto a las pacientes resistentes al tratamiento con depresión mayor postnatal de gravedad leve a moderada, pueden no responder al tratamiento inicial y la respuesta normalmente es una reducción de los síntomas de referencia de más de un 50 por ciento (19).

\section{DEPRESIÓN POSTPARTO SEVERA}

Las pacientes gravemente enfermas suelen reportar la ideación y el comportamientos suicidas, por lo general demuestran un deterioro obvio del funcionamiento y a menudo manifiestan un mal juicio, que coloca al paciente y a otros (incluido el recién nacido y demás niños a cargo de la paciente), en riesgo de daño inminente.

También, es más probable que las pacientes con depresión posparto severa desarrollen complicaciones tales como rasgos psicóticos y rasgos catatónicos.

Las pacientes con depresión severa deben ser referidas a un psiquiatra para el manejo y a menudo requiere hospitalización, para tratar la depresión mayor con rasgos psicóticos o catatónicos, su tratamiento no se discutirá en este artículo (28).

Respecto al manejo de las pacientes con depresión posparto severa, la psicoterapia casi siempre se indica como un adyuvante a la farmacoterapia (que se basa en la utilización de medicamentos antidepresivos) y se recomienda que reciban psicoterapia inicial y se siga evaluando su progreso hasta que respondan.

Además, para las pacientes con este tipo de depresión que se encuentran amamantando, se sugieren medicamentos antidepresivos en base a su eficacia en ensayos aleatorios, ya que estos están más disponibles en la práctica médica y su uso es más beneficioso en combinación de pautas en la práctica múltiple con psicoterapia (27).

\section{- Farmacoterapia}

Existe un consenso general de que los beneficios de los antidepresivos superan los riesgos potenciales para el bebé, considerando que son típicamente bajos $(27,29,30)$.

La evidencia indirecta que apoya el uso de antidepresivos para la depresión postparto incluye varios ensayos aleatorios que excluyeron a las pacientes que estaban amamantando. Estos ensayos han demostrado que numerosos antidepresivos (p. ej., inhibidores selectivos de la recaptación de serotonina (ISRS) e inhibidores de la recaptación de norepinefrina (bupropión y mirtazapina); pueden se utilizados eficazmente a la población general de pacientes con depresión severa y la población de madres con este tipo de depresión se pueden ver beneficiadas $(31,32)$. 
Dentro de los antidepresivos más usados y con mayor evidencia se encuentran los ISRS, de elección la paroxetina o sertralina para el tratamiento inicial; debido a que los efectos adversos en los lactantes han demostrado ser más bajos; adémas los estudios sugieren que la paroxetina y la sertralina, son generalmente indetectables en sangre de los lactantes que se exponen a través de la leche materna. Sin embargo, el citalopram también es un alternativa razonable (27).

\section{CONCLUSIÓN}

La depresión posparto es una de las complicaciones más comunes en el puerperio, por ende es fundamental la detección clínica temprana de esta patología; a partir de una evaluación integral de los factores de riesgo a nivel familiar y social; como lo es la violencia intrafamiliar, drogadicción, conflictos de pareja, problemas legales, entre otros. Además de valorar antecedentes psiquiátricos de depresión posparto previos $\mathrm{y} / \mathrm{o}$ otros trastornos psiquiátricos como la bipolaridad.

Debido a que todos estos antecedentes patológicos en la madre, aumentan el riesgo de cursar nuevamente con episodios depresivos de mayor intensidad durante el puerperio, ansiedad, ataques de pánico y episodios con características psicóticas.

La sospecha de esta patología por parte del personal de salud, es de suma importancia para poder reconocer clínicamente este trastorno; que a pesar de no cumplir con todos los criterios de diagnóstico como tal; se logre la prevención de madres con inicio de los síntomas. Esto con el fin de evitar posibles complicaciones que impliquen trastornos mentales en la madre, en el proceso de lactancia, apego entre la madre y el producto, así como problemas de comportamiento y desarrollo del niño a futuro. También como parte del correcto diagnóstico, se debe diferenciar otras patologías de origen orgánico como hipotiroidismo, anemia por deficiencia de hierro y otros trastornos de ánimo; como posibles causas subyacentes e instaurar su respectivo tratamiento en cada caso.

Respecto al manejo terapéutico, tomando a la psicoterapia como pilar principal para todos los diferentes niveles de severidad, se recomienda la psicoterapia como opción de primera línea de tratamiento para mujeres con depresión posparto de leve a moderada, siempre valorando su progreso y respuesta, para depresiones de moderadas a severas, asociar la psicoterapia al uso de antidepresivos aprobados para el período de lactancia; debido a que los beneficios de los antidepresivos superan los riesgos potenciales para el bebé, en este tipo de depresión. Pacientes con síntomas graves, como ideación suicida coloca a la paciente y a otros (incluido el recién nacido y demás niños a cargo de la paciente), en riesgo de daño inminente. Por lo que se aconseja que en este tipo de casos, deban ser referidos al psiquiatra lo antes posible, así como tomar medidas de protección tanto para la madre como para su recién nacido o demás hijos y valorar su hospitalización. Debido a que este trastorno es un desorden potencialmente devastador que puede sobrellevar grandes consecuencias en la vida de la mujer y sus hijos. 


\section{AGRADECIMIENTOS}

En agradecimiento a la colaboración y ayuda brindada de las Doctoras: Sofía de los Ángeles Mora Agüero y Mariam Lucía Soto Solís.

\section{REFERENCIAS}

1. Gavin NI, Gaynes BN, Lohr KN, Meltzer-Brody S, Gartlehner G, Swinson T. Perinatal depression: a systematic review of prevalence and incidence. Obstet Gynecol. 2005; 106 (5 Pt 1): 10711083. https://doi.org/10.1097/01.AOG.0000183597.31630.db

2. DeCherney, Alan H Diagnostico y tratamiento ginecoobstetricos. 11ed. Mexico: McGraw Hill; 2014. 367$368 \mathrm{p}$.

3. Epperson CN. Postpartum major depression: detection and treatment. Am Fam Physician. 1999; 59(8): 2247-2254.

4. American Psychiatric Association. Diagnostic and statistical manual of mental disorders. 5th ed. 2. Arlington: American Psychiatric Association; 2014. 123-125 p.

5. Logsdon MC, Wisner KL, Pinto-Foltz MD. The impact of peripartum depression on mothering. J Obstet Gynecol Neonatal Nurs . 2006; 35(5): 652-658. https://doi.org/10.1111/j.1552-6909.2006.00087.x

6. Nock M, Borges G, Bromet E, Cha C, Kessler R, Lee S. Suicide and suicidal behavior. EpidemiolRev. 2008; 30(1): 133-154. https://doi.org/10.1093/epirev/mxn002

7. Friedman S, Hrouda D, Holden C, Noffsinger S, Resnick P. Filicide-suicide: common factors in parents who kill their children and themselves. J Am Acad Psychiatry Law. 2005; 33(4): 496-504.

8. Guille C, Newman R, Fryml LD, Lifton CK, Epperson CN. Management of postpartum depression. J Midwifery Womens Health. 2013; 58(6): 643-653.https://doi.org/10.1111/jmwh.12104

9. Borget D, Grace J, Whitehurst L. A review of maternal and paternal filicide. J Am Acad Psychiatry Law. 2007; 35(1): 74-82

10. Shariatpanaahi MV, Shariatpanaahi ZV, Moshtaaghi M, Shahbaazi SH, Abadi A. The relationship between depression and serum ferritin level. Eur J Clin Nutr. 2007; 61 (4): 532535.http://dx.doi.org/10.1038/sj.ejcn.1602542

11. Corwin EJ, Murray-Kolb LE, Beard JL. Low hemoglobin level is a risk factor for postpartum depression. J Nutr. 2003; 133 (12): 4139-4142. https://doi.org/10.1093/jn/133.12.4139

12. Bodnar LM, Siega-Riz AM, Miller WC, Cogswell ME, McDonald T. Who should be screened for postpartum anemia? An evaluation of current recommendations. Am J Epidemiol 2002; 156 (10): 903912. https://doi.org/10.1093/aje/kwf134

13. Murray-Kolb LE, Beard JL. Iron deficiency and child and maternal health. Am J Clin Nutr 2009;89(3) :946S-950S. https://doi.org/10.3945/ajcn.2008.26692D

14. Ludwig H, Strasser K. Symptomatology of anemia. Semin Oncol. 2001; 28(Suppl 8): 7 14.https://doi.org/10.1016/S0093-7754(01)90206-4

15. Myint AM, Leonard BE, Steinbusch HW, Kim YK. Th1, Th2, and Th3 cytokine alterations in major depression. J Affect Disord. 2005 Oct; 88(2): 167-173.https://doi.org/10.1016/j.jad.2005.07.008

16. Sines E, Uzma S, Wall S, Worley H. Postnatal care: A critical opportunity to save mothers and newborns [Internet]. Policy Perspectives on Newborn Health-Saving Newborns Lives. Washington: Population reference $\quad$ bureau. 2007 15-02-2019]. Disponible en:https://www.researchgate.net/publication/242720964 Postnatal Care A Critical Opportunity to Save Mothers and Newborns 
17. Yonkers KA, Vigod S, Ross LE. Diagnosis, pathophysiology, and management of mood disorders in pregnant and postpartum women. Obstet Gynecol. 2011 Apr; 117(4): 96177.https://doi.org/10.1097/AOG.0b013e31821187a7

18. Putnam K, Robertson-Blackmore E, Sharkey K, Payne J, Bergink V, Munk-Olsen T, et al. Heterogeneity of postpartum depression: a latent class analysis. Lancet Psychiatry. 2015 Jan; 2(1): 5967. https://doi.org/10.1016/S2215-0366(14)00055-8

19. Stein A, Pearson RM, Goodman SH, Rapa E, Rahman A, McCallum M, et al. Effects of perinatal mental disorders on the fetus and child. Lancet. 2014 Nov 15; 384(9956): 18001819.https://doi.org/10.1016/S0140-6736(14)61277-0

20. Larsen ER, Damkier P, Pedersen LH, Fenger-Gron J, Mikkelsen RL, Nielsen RE, et al. Use of psychotropic drugs during pregnancy and breast-feeding. Acta Psychiatr Scand Suppl. 2015; 132(S445). https://doi.org/10.1111/acps.12479

21. Lynch D, Laws KR, McKenna PJ. Cognitive behavioural therapy for major psychiatric disorder: does it really work? A meta-analytical review of well-controlled trials. Psychol Med. 2010 Jan; 40(1): 924. https://doi.org/10.1017/S003329170900590X

22. Barth J, Munder T, Gerger H, Nüesch E, Trelle S, Znoj H, et al. Comparative efficacy of seven psychotherapeutic interventions for patients with depression: a network meta-analysis. PLoS Med. 2013 ;10(5): e1001454. https://doi.org/10.1371/journal.pmed.1001454

23. Miniati M, Callari A, Calugi S, Rucci P, Savino M, Mauri M, et al. Interpersonal psychotherapy for postpartum depression: a systematic review. Arch Womens Ment Health. 2014 Aug; 17(4): 257268. https://doi.org/10.1007/s00737-014-0442-7

24. Dennis CL. Psychosocial interventions for the treatment of perinatal depression. Best Pract Res Clin Obstet Gynaecol. 2014 Jan; 28(1): 97-111.https://doi.org/10.1016/j.bpobgyn.2013.08.008

25. Cooper PJ, Murray L, Wilson A, Romaniuk H. Controlled trial of the short- and long-term effect of psychological treatment of post-partum depression. I. Impact on maternal mood. Br J Psychiatry. 2003 May; 182: 412-419. https://doi.org/10.1192/bjp.182.5.412

26. Scope A, Leaviss J, Kaltenthaler E, Parry G, Sutcliffe P, Bradburn M, et al. Is group cognitive behaviour therapy for postnatal depression evidence-based practice? A systematic review. BMC Psychiatry. 2013 Nov 28;13:321. https://doi.org/10.1186/1471-244X-13-321

27. O'Hara MW, McCabe JE. Postpartum depression: current status and future directions. Annu Rev Clin Psychol. 2013; 9: 379-407. https://doi.org/10.1146/annurev-clinpsy-050212-185612

28. Fink M, Taylor MA. The catatonia syndrome: forgotten but not gone. Arch Gen Psychiatry. 2009 Nov; 66(11): 1173-1177. https://doi.org/10.1001/archgenpsychiatry.2009.141

29. Meltzer-Brody S, Jones I. Optimizing the treatment of mood disorders in the perinatal period. Dialogues Clin Neurosci. 2015 Jun;17(2):207-218.

30. Kim DR, Epperson CN, Weiss AR, Wisner KL. Pharmacotherapy of postpartum depression: an update. Expert Opin Pharmacother. 2014 Jun; 15(9): 1223-1234.https://doi.org/10.1517/14656566.2014.911842

31. Singh SP, Singh V, Kar N. Efficacy of agomelatine in major depressive disorder: meta-analysis and

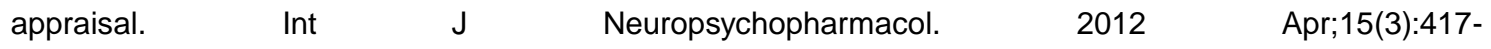
428.https://doi.org/10.1017/S1461145711001301

32. Milgrom J, Gemmill AW, Ericksen J, Burrows G, Buist A, Reece J. Treatment of postnatal depression with cognitive behavioural therapy, sertraline and combination therapy: a randomised controlled trial. Aust N Z J Psychiatry. 2015 Mar; 49(3): 236-245.https://doi.org/10.1177/0004867414565474 